\title{
Statistical strained-tetrahedron model of local ternary zinc blende crystal structures
}

\author{
B.V. Robouch ${ }^{1}$, E.M. Sheregii ${ }^{2}$, and A. Kisiel ${ }^{3}$ \\ ${ }^{1}$ Laboratori Nazionali di Frascati INFN, DAFNE-L, C.P.:13, 00044 Frascati (RM), Italy \\ E-mail: Robouch@lnf.infn.it \\ ${ }^{2}$ Institute of Physics, Rzeszow University, Rejtana 16A, 35-310 Rzeszow, Poland \\ ${ }^{3}$ Instytut Fizyki, Universytet Jagiellonski, Reymonta 4, 30-059 Krakow, Poland
}

Received June 1, 2004

\begin{abstract}
The statistical strained-tetrahedron model was developed to overcome two common assumptions of previous models: 1) rigid undistorted ion sublattice of regular tetrahedra throughout all five configurations and 2) random ion distribution. These simplifying assumptions restrict the range of applicability of the models to a narrow subset of ternary alloys for which the constituent binaries have their lattice constants and standard molar enthalpies of formation $\left(\Delta_{f} H_{0}\right)$ equal or quasi-equal. Beyond these limits predictions of such models become unreliable, in particular, when the ternary exhibits site occupation preferences. The strained-tetrahedron model, free from rigidity and stochastic limitations, was developed to better describe and understand the local structure of ternary zinc blende crystals, and interpret experimental EXAFS and far-IR spectra. It considers five tetrahedron configurations with the shape and size distortions characteristic of ternary zinc blende alloys, allows nonrandom distributions and, hence, site occupation preferences, conserves coordination numbers, respects stoichiometry, and assumes that next-neighbor values determine preferences beyond next-neighbor. The configuration probabilities have three degrees of freedom. The nineteen inter-ion crystal distances are constrained by tetrahedron structures; to avoid destructive stresses, we assume that the average tetrahedron volumes of both sublattices relax to equal values. The number of distance free-parameters $\leq 7$. Model estimates, compared to published EXAFS results, validate the model. Knowing the configuration probabilities, one writes the dielectric function for far-infrared absorption or reflection spectra. Constraining assumptions restrict the number of degrees of freedom. Deconvolution of the experimental spectra yields site-occupation-preference coefficient values and/or specific oscillator strengths. Validation again confirms the model.
\end{abstract}

PACS: 78.30.Er

\section{Introduction}

The abundance of articles in the literature devoted to sphalerite (zinc blende) ternary semiconductors is ample evidence of the interest paid to them. In the hope of better understanding their local structure, we considered the interpretation of extended x-ray absorption fine structure (EXAFS) (see theoretical considerations [1,2] since 1981) and vibrational spectra observed in the far-infrared region (FIR spectra). EXAFS was applied soon after [3,4] with, alas, no review paper covering the abundant literature devoted to it. For literature on the FIR spectra, see, for instance, the review articles [5-7] and book [8]. With that aim we developed the statistical strained-tetrahedron model, validating it on published EXAFS zinc blende data $[9,10]$ and, after an adaptation, on intermetallide materials [11]. The model was then extended to describe and interpret FIR spectra [12].

We propose to briefly recall here the model developed and its validation and then to dwel more on FIR-spectrum interpretation, applicability, and limits.

\section{The statistical strained-tetrahedron model}

To create any model, one has to 1) describe as closely as possible the object under study, using 
proper parameters for that; 2) reduce the set of these parameters through motivated constraints to determine the minimum number of degrees of freedom / $\mathrm{pa}-$ rameters; 3 ) check the model-predicted values against experimental ones; 4) consequently, discard the model or retain it as valid depending on the reproducibility thus obtained. With this in mind we recall our modeling.

\subsection{The object under study}

Zinc blende fcc structures are tetrahedrally coordinated, characterized by a central ion surrounded by four nearest-neighbor (NN) ions (first shell) defining the four vertices of a tetrahedron, and 12 next nearest-neighbor (NNN) ions (second shell). Binary compounds $\mathrm{AZ}$ (we use $\mathrm{A}, \mathrm{B}, \ldots$ for cations, and $\mathrm{Z}, \mathrm{Y}, \ldots$ for anions), have their successive shells alternately fully filled by $\mathrm{A}$ then $\mathrm{Z}$ ions. All tetrahedra are symmetric, regular, and identical; thus, by simple trigonometry, equal interbond angles $\alpha(\mathrm{A}: \mathrm{Z}: \mathrm{A})=$ $=\alpha(\mathrm{B}: \mathrm{Z}: \mathrm{A})=109.47^{\circ}$, and the inter-ion distances $\left({ }^{i j} d\right)$ are defined in terms of the lattice constant $a$ (known from x-ray diffraction analysis): ${ }^{\mathrm{AZ}} d=3^{1 / 2} a / 4$, ${ }^{\mathrm{AA}} d={ }^{\mathrm{ZZ}} d=a / 2^{1 / 2}$.

For ternary $\mathrm{A}_{1-x} \mathrm{~B}_{x} \mathrm{Z}$ (or $\mathrm{AY}_{y} \mathrm{Z}_{1-y}$ ), in the binary compound $\mathrm{AZ}$, cations $\mathrm{A}$ are partially substituted by $\mathrm{B}$ ions. This, leads to five different elemental tetrahedra $\left\{T_{k}\right\}_{k=0,4}$ where the subscript $k$ indicates the number of $\mathrm{B}$ ions at the vertices of the tetrahedron, with (4-k) A-ions $\left[T_{0}\left(\mathrm{Z}:{ }^{4} \mathrm{~A}\right), T_{1}\left(\mathrm{Z}:{ }^{3} \mathrm{~A}+{ }^{1} \mathrm{~B}\right), T_{2}\left(\mathrm{Z}:{ }^{2} \mathrm{~A}+{ }^{2} \mathrm{~B}\right)\right.$, $\left.T_{3}\left(\mathrm{Z}:{ }^{1} \mathrm{~A}+{ }^{3} \mathrm{~B}\right), T_{4}\left(\mathrm{Z}:{ }^{4} \mathrm{~B}\right)\right]$. Prior to ours, simulations had considered the five $\left\{T_{k}\right\}_{k=0,4}$ tetrahedra as externally rigid with the central ion free to be displaced. The ion distribution fillings ( $k$ B-ions into a shell with $N$ sites, from relative contents $x$ and $1-x$ ) were assumed stochastic and defined by the random Bernoulli binomial polynomials

$p_{k}{ }^{[N]}(x)=N ! /[k !(N-k) !] x^{k}(1-x)^{N-k}$ with $k=0, \ldots, N$.

Thus around a central $\mathrm{Z}$ ion the first shell four $\mathrm{A} / \mathrm{B}$ ions are described by $p_{k}^{[4]}(x)$, while the second shell contains twelve $\mathrm{Z}$ ions! On the other hand, around an A or B ions, the first shell contains four $\mathrm{Z}$ ions, while the second shell distribution of the twelve ions $\mathrm{A} / \mathrm{B}$ is described by $p_{k}{ }^{[12]}(x)$. This allows approximate evaluations, avoiding analytical difficulties.

However, to assume a stochastic filling with ions A and $\mathrm{B}$ around $\mathrm{Z}$ means that the $\mathrm{Z}$-ion preference for either is the same. Thermodynamically this implies that the enthalpies of formation of $\mathrm{AZ}$ and $\mathrm{BZ}$ pairs are identical. But we are aware that in nature equality is the exception that confirms the rule of inequality. Indeed, the standard molar enthalpies of formation of bi- naries, $\Delta_{f} H_{0}, \mathrm{~kJ} / \mathrm{mol}$, are generally different. That is why the stochastic approach is unable to describe the site occupation preferences (SOPs) reported experimentally!

\subsection{Statistical strained-tetrahedron model assumptions}

We build our model discarding both restrictions: 1) deviating from stochastic filling of ions, and 2) freeing the tetrahedra of the unnatural constraint of rigidity.

The price for such a more general model is the number of parameters needed to describe the crystal structure. But as we shall demonstrate, realistic assumptions (checked at the end) reduce the degrees of freedom to an acceptable value.

To quantify results departing from stochastic distribution, we attribute to each Bernoulli binomial a SOP weight coefficient. This leads to five NN terms $W_{k} p_{k}{ }^{[4]}(x)$, thirteen NNN terms ${ }^{\mathrm{A}_{w}}{ }_{k} p_{k}{ }^{[12]}(x)$ (for central A ions), and thirteen ${ }^{\mathrm{B}_{w_{k}}} p_{k}{ }^{[12]}(x)$ terms (for central B ions), a total of 31 parameters! Fortunately it is the $\mathrm{Z}$ ion that determines the choices, and we claim that ALL higher shell fillings are determined by linear expressions of the five NN SOP coefficients $\left\{W_{k}\right\}_{k=0,4}$. But binary tetrahedron configurations $T_{0}$, $T_{4}$ have NO preferences. Thus $W_{0}=W_{4} \equiv 1$ (!) and we are left with only $\left\{W_{1}, W_{2}, W_{3}\right\}$.

The probabilities of finding $\mathrm{B}$ and $\mathrm{A}$ ions in a $T_{k}$ configuration are proportional to $\left\{k W_{k}\right\}_{k=0,4}$ and to $\left\{4-k W_{k}\right\}_{k=0,4}$, respectively (conservation of coordination numbers). As probabilities cannot be negative, we have $0 \leq W_{k} \leq 4 / k$. There are thus only three bounded free parameters $\left\{W_{1}, W_{2}, W_{3}\right\}$.

Expressions $\left\{{ }^{\mathrm{A}} w_{k}\right\}_{k=0,12},\left\{{ }^{\mathrm{B}} w_{k}\right\}_{k=0,12}$ for the NNN shell, are determined by combinatorial probabilities in terms of those around the $\mathrm{Z}$ ions of the $\mathrm{NN}$ shell. This hypothesis leads for the zinc blende structure, to the linear expressions of the NNN SOP coefficients ${ }^{\mathrm{A}} w_{k}$ and $\mathrm{B}_{w_{k}}$ as functions of the three $W_{k}$ 's given in Table 1, $a$ [9]. To illustrate that the assumption is general and applies to other crystal structures also, the expressions for intermetalides $\mathrm{M}_{3}\left(\mathrm{X}_{1-x} \mathrm{X}_{x}^{\prime}\right)_{1}$ are given in Table 1,b [11].

A random integer ion distribution ( $k$ and $4-k$ ) fully respects stoichiometry. With SOP coefficients $W_{k} \neq 1$ the situation departures from stochastic equilibrium, with a consequent attenuation of the ternary configuration populations caused by the observed scarcity of one of the two ion populations

$$
\left\{P_{k}^{[4]}(x)=C_{k} p_{k}{ }^{[4]}(x)\right\}_{k=1,3} \text { for ternary } T_{k}
$$

with $\left\{C_{k}\left(W_{k}\right)\right\}_{k=1,3}$, corrective weight factors imposed by stoichiometry 
NNN SOP-coefficients in terms of NN SOPs: All possible NNN distributions and resulting SOP-coefficients for ternary. $0 \leq\left\{W_{k}\right\}_{k=1,3} \leq 4 / k$, while $W_{0}=W_{4}=1$. Zinc blende $\mathrm{A}_{1-x} \mathrm{~B}_{x} \mathrm{Z}$ with a $\mathrm{B}$ or $\mathrm{A}$ as central ion [9].

\begin{tabular}{|c|c|c|c|}
\hline$k$ & $\begin{array}{c}\text { All } 12 \text { possible NNN B-ion fills } \\
\text { of the } 4 \text { tetrahedra }\end{array}$ & $\begin{array}{l}\text { Resulting B-weights } \\
{ }_{\mathrm{B}^{\mathrm{B}}{ }_{k}{ }_{k}^{[12]}=\sum_{j=0,4}\left\{{ }^{\mathrm{B}} M_{j, k} W_{j}^{[4]}\right\}}\end{array}$ & 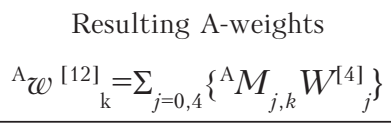 \\
\hline 0 & $\begin{array}{llllllllll}0 & 0 & 0 & 0\end{array}$ & $W_{1}$ & $W_{0}=1$ \\
\hline 1 & 0001 & $\left(3 W_{1}+W_{2}\right) / 4$ & $\left(3 W_{0}+W_{1}\right) / 4$ \\
\hline 2 & $00002 / 0011$ & $\left(5 W_{1}+2 W_{2}+W_{3}\right) / 8$ & $\left(5 W_{0}+2 W_{1}+W_{2}\right) / 8$ \\
\hline 3 & $0003 / 0012 / 0111$ & $\left(6 W_{1}+4 W_{2}+W_{3}+W_{4}\right) / 12$ & $\left(6 W_{0}+4 W_{1}+W_{2}+W_{3}\right) / 12$ \\
\hline 4 & $0013 / 0022 / 0112 / 11111$ & $\left(5 W_{1}+7 W_{2}+3 W_{3}+W_{4}\right) / 16$ & $\left(5 W_{0}+7 W_{1}+3 W_{2}+W_{3}\right) / 16$ \\
\hline 5 & $0023 / 0113 / 0122$ & $\left(4 W_{1}+3 W_{2}+3 W_{3}+2 W_{4}\right) / 12$ & $\left(4 W_{0}+3 W_{1}+3 W_{2}+2 W_{3}\right) / 12$ \\
\hline 6 & $0033 / 0123 / 1113 / 0222 / 1122$ & $\left(4 W_{1}+6 W_{2}+6 W_{3}+4 W_{4}\right) / 20$ & $\left(4 W_{0}+6 W_{1}+6 W_{2}+4 W_{3}\right) / 20$ \\
\hline 7 & $0133 / 0223 / 1123$ & $\left(2 W_{1}+3 W_{2}+3 W_{3}+4 W_{4}\right) / 12$ & $\left(2 W_{0}+3 W_{1}+3 W_{2}+4 W_{3}\right) / 12$ \\
\hline 8 & $0233 / 1223 / 1133 / 2222$ & $\left(W_{1}+3 W_{2}+7 W_{3}+5 W_{4}\right) / 16$ & $\left(W_{0}+3 W_{1}+7 W_{2}+5 W_{3}\right) / 16$ \\
\hline 9 & $0333 / 1233 / 2223$ & $\left(W_{1}+W_{2}+4 W_{3}+6 W_{4}\right) / 12$ & $\left(W_{0}+W_{1}+4 W_{2}+6 W_{3}\right) / 12$ \\
\hline 10 & $1333 / 2233$ & $\left(W_{2}+2 W_{3}+5 W_{4}\right) / 8$ & $\left(W_{1}+2 W_{2}+5 W_{3}\right) / 8$ \\
\hline 11 & 2333 & $\left(W_{3}+3 W_{4}\right) / 4$ & $\left(W_{2}+3 W_{3}\right) / 4$ \\
\hline 12 & 3333 & $W_{4}=1$ & $W_{3}$ \\
\hline
\end{tabular}

Table $1, b$

Intermetallides $\mathrm{M}_{3}\left(\mathrm{XX}^{\prime}\right)_{1}$ around $\mathrm{X}$ or $\mathrm{X}^{\prime}$ [11].

\begin{tabular}{|c|c|}
\hline & $\left\{\mathrm{X}_{w_{k}}=\Sigma_{j=0,4} \mathrm{X}_{M_{k j}} W_{j}\right\}_{k=0,6}$ \\
\hline$x_{w_{0}}=$ & $1 / 3 W_{1}+2 / 3 W_{2}$ \\
\hline$x_{w_{1}}=$ & $10 / 36 W_{1}+23 / 36 W_{2}+3 / 36 W_{3}$ \\
\hline$x_{w_{2}}=$ & $16 / 72 W_{1}+41 / 72 W_{2}+13 / 72 W_{3}+2 / 72 W_{4}$ \\
\hline$x_{w_{3}}=$ & $6 / 36 W_{1}+15 / 36 W_{2}+11 / 36 W_{3}+4 / 36 W_{4}$ \\
\hline$x_{w_{4}}=$ & $2 / 72 W_{1}+13 / 72 W_{2}+41 / 72 W_{3}+16 / 72 W_{4}$ \\
\hline$x_{w_{5}}=$ & $3 / 36 W_{2}+23 / 36 W_{3}+10 / 36 W_{4}$ \\
\hline$x_{w_{6}}=$ & $+2 / 3 W_{3}+1 / 3 W_{4}$ \\
\hline
\end{tabular}

$$
0 \leq\left\{C_{k}=\min \left[W_{k}, 1,\left(4-k W_{k}\right) /(4-k)\right]\right\}_{k=1,3} \leq 1
$$
$W_{k}<1$ enhances the binary AZ populations and $W_{k}>1$ that of binary BZ, i.e.

$$
P_{0}^{[4]}(x)=p_{0}{ }^{[4]}(x)+\sum_{k=1,3}\left\{\max \left(0,1-W_{k}\right) p_{k}{ }^{[4]}(x)\right\}
$$

for binary AZ configuration $T_{0}$,

$$
\begin{gathered}
P_{4}{ }^{[4]}(x)=p_{4}{ }^{[4]}(x)+ \\
+\sum_{k=1,3}\left\{\max \left(0, k\left(W_{k}-1\right) /(4-k)\right) p_{k}(x)\right\}
\end{gathered}
$$

for binary BZ configuration $T_{4}$.

In the random case, when $\left\{W_{k} \equiv 1\right\}_{k=1,3},\left\{P_{k}(x) \rightarrow\right.$ $\left.\rightarrow p_{k}(x)\right\}_{k=0,4}$.

\begin{tabular}{cc}
\hline \hline & $\left\{{ }^{X^{\prime}} w_{k}=\sum_{j=0,4}{ }^{\prime}{ }^{\prime} M_{k j} W_{j}\right\}_{k=0,6}$ \\
$X^{\prime} w_{0}=$ & $1 / 3 W_{0}+2 / 3 W_{1}$ \\
$X^{\prime} w_{1}=$ & $10 / 36 W_{0}+23 / 36 W_{1}+3 / 36 W_{2}$ \\
$X^{\prime} w_{2}=$ & $16 / 72 W_{0}+41 / 72 W_{1}+13 / 72 W_{2}+2 / 72 W_{3}$ \\
$X^{\prime} w_{3}=$ & $6 / 36 W_{0}+15 / 36 W_{1}+11 / 36 W_{2}+4 / 36 W_{3}$ \\
$X^{\prime} w_{4}=$ & $2 / 72 W_{0}+13 / 72 W_{1}+41 / 72 W_{2}+16 / 72 W_{3}$ \\
$X^{\prime} w_{5}=$ & $3 / 36 W_{1}+23 / 36 W_{2}+10 / 36 W_{3}$ \\
$X^{\prime} w_{6}=$ & $+2 / 3 W_{2}+1 / 3 W_{3}$ \\
\hline \hline
\end{tabular}

Note however, that even if the SOPs enhance the two binary populations with respect to corresponding populations of the random case, it by no means leads to clustering, since the spatial distribution remains perfectly stochastic.

Ion-pair and configuration populations are NOW determined with due account of the SOPs. This allows us to interpret local crystal structures.

We have five tetrahedra freed from any constraint, with two of which $\left(T_{0}\right.$ and $\left.T_{4}\right)$ are binary, regular, different sized, and well defined (as remarked above), and three $\left(T_{1}, T_{2}\right.$, and $\left.T_{3}\right)$ are ternary and distorted (strained tetrahedra), with nineteen unequal interionic ${ }^{i j} d$ distance parameters and, consequently, altered interbond angles (see Fig. 1 [9]). The geometrical symmetry of each $T_{k}$ configuration yields three 


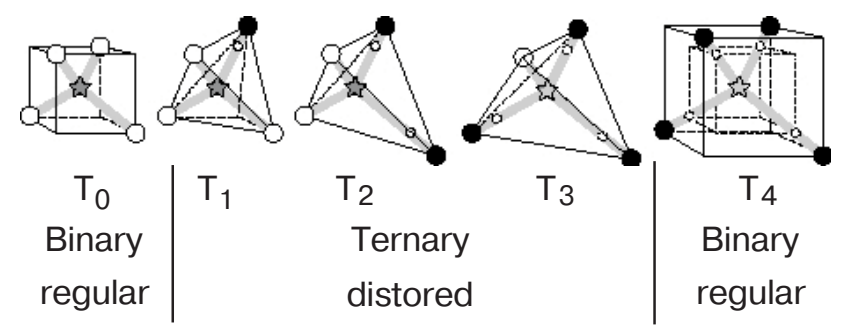

Fig. 1. Aspect of the five elemental tetrahedron configurations $\left\{T_{k}\right\}_{k=0,4}$ of $\mathrm{A}_{1-x} \mathrm{~B}_{x} \mathrm{Z}$ (or $\mathrm{AY}_{y} \mathrm{Z}_{1-y}$ ) ternary alloys [9]. Small open circles indicate the would-be ion-positions as per rigid tetrahedron hypothesis.

constraints each, which reduces the number of independent distance parameters from nineteen to ten.

Material strength considerations lead us, to avoid destructive intercrystal stresses, to impose the condition that the average tetrahedron volume of the four vertex tetrahedra be equal to the central one (one constraint per configuration), leaving us with only
$(10-3)=7$ distance parameters, while for SOP extreme values, configurations disappear, and their distances become virtual, i.e., $\leq 7$.

Indeed $\leq$, as for extreme SOP values configurations disappear, and their distances become virtual!

On the basis of the above probabilities, expressions for the average pair coordination $\left\langle{ }^{i: j} \mathrm{CN}(x)>\right.$ and distances $<^{i: j} d(x)>$ as functions of $x$, for any two-ion pair $i: j=\{\mathrm{AZ}, \mathrm{BZ}, \mathrm{BB}, \mathrm{BA}, \mathrm{AA}, \mathrm{ZAZ}, \mathrm{ZBZ}\}$ of zinc blende ternary alloys $\mathrm{A}_{1-x} \mathrm{~B}_{x} \mathrm{Z}$ are given in Table 2 [9].

On the basis of these, deconvolving a given set of EXAFS data such as $\mathrm{GaAs}_{y} \mathrm{P}_{1-y}$ [13], one obtains the dimensions of all the elemental tetrahedra involved: the inter-ion distances and angles (see Table 3 ).

Having defined a 31+19 parameter model and identified the relative constraint relations, we have reduced the problem to $3+7$ independent parameters. The model is ready for confrontation of its estimations with experimental data.

Table 2

Expressions of average pair coordinations $\left\langle{ }^{i: j} \mathrm{CN}(x)>\right.$ and distances $<^{i: j} d(x)>$, as a function of $x$, for any two-ion pair $i: j=$ $=\{\mathrm{AZ}, \mathrm{BZ}, \mathrm{BB}, \mathrm{BA}, \mathrm{AA}, \mathrm{ZAZ}, \mathrm{ZBZ}\}$ of zinc blende $\mathrm{A}_{1-x} \mathrm{~B}_{x} \mathrm{Z}$ ternary $[9]$.

\begin{tabular}{|c|c|}
\hline Average & $\begin{array}{c}\text { Coordination numbers } \\
\text { for NN ion pairs AZ, BZ }\end{array}$ \\
\hline $\begin{array}{l}<{ }^{\mathrm{BZ}} \mathrm{CN}(x)>= \\
<{ }^{\mathrm{AZ}} \mathrm{CN}(x)>=\end{array}$ & $\begin{array}{l}\Sigma_{k=0,4}\left\{k W_{k} p_{k}^{[4]}(x)\right\} \\
\Sigma_{k=0,4}\left\{\left(4-k W_{k}\right) p_{k}^{[4]}(x)\right\}=4-<{ }^{\mathrm{BZ}} \mathrm{CN}(x)>\end{array}$ \\
\hline & For NNN ion pairs BB, BA, AA \\
\hline $\begin{array}{l}<{ }^{\mathrm{BB}} \mathrm{CN}(x)>= \\
<{ }^{\mathrm{AB}} \mathrm{CN}(x)>= \\
<{ }^{\mathrm{AA}} \mathrm{CN}(x)>= \\
<{ }^{\mathrm{BA}} \mathrm{CN}(x)>= \\
<{ }^{\mathrm{ZBZ}} \mathrm{CN}(x)>= \\
<{ }^{\mathrm{ZAZ}} \mathrm{CN}(x)>=\end{array}$ & 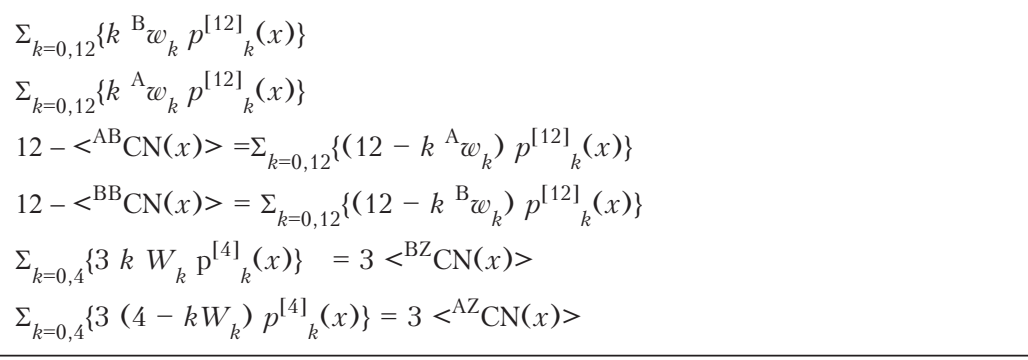 \\
\hline Average & $\begin{array}{c}\text { Distances } \\
\text { for NN ion pairs AZ, BZ }\end{array}$ \\
\hline$<{ }^{\mathrm{BZ}} d(x)>=$ & $\begin{array}{ccc}\left\{\Sigma_{k=1,4}\left\{k C_{k}{ }^{\mathrm{BZ}} d_{k}\right.\right. & \left.\left.+4 \operatorname{Max}\left[0, k\left(W_{k}-1\right) /(4-k)\right]{ }^{\mathrm{BZ}} d_{4}\right\} p^{[4]}{ }_{k}(x)\right\} \\
/\left\{\Sigma_{k=1,4}\left\{k C_{k}\right.\right. & +4 \operatorname{Max}\left[0, k\left(W_{k}-1\right) /(4-k)\right] & \left.\} p^{[4]}(x)\right\} \\
\left\{\Sigma_{k=0,3}\left\{\left(4-k C_{k}\right){ }^{\mathrm{AZ}} d_{k}\right.\right. & +4 \operatorname{Max}\left[0,\left(1-W_{k}\right)\right] & \left.\left.\mathrm{AZ}_{d_{0}}\right\} p_{k}^{[4]}(x)\right\} \\
/\left\{\Sigma_{k=0,3}\left\{\left(4-k C_{k}\right)\right.\right. & +4 \operatorname{Max}\left[0,\left(1-W_{k}\right)\right] & \left.\} p_{k}^{[4]}(x)\right\}\end{array}$ \\
\hline & For NNN ion pairs Z:A:Z or Z:B:Z \\
\hline$<^{\mathrm{ZAZ}} d(x)>=$ & 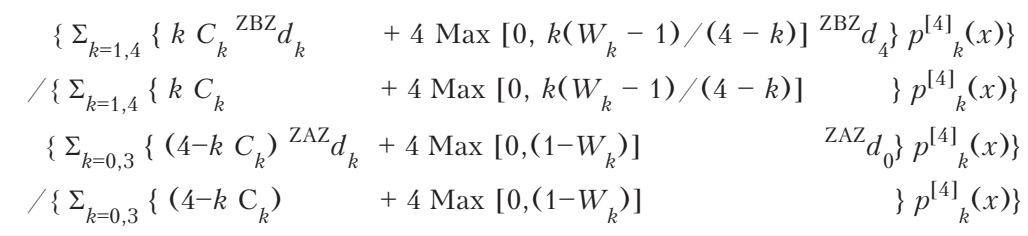 \\
\hline
\end{tabular}


Table 3

$\mathrm{GaAs}_{x} \mathrm{P}_{1-x}$ complete set: determined SOP coefficients, distances, angles and volumes for all five elemental configuration tetrahedra. Eleven fit parameters (bold) (3-SOP + 8-distance (PGaP NNN data not reported) to check VRC. 37 available experimental points from a set of 16 measurements [9].

\begin{tabular}{|c|c|c|c|c|c|}
\hline Configurations & $T_{0}$ & $T_{1}$ & $T_{2}$ & $T_{3}$ & $T_{4}$ \\
\hline$k$ & 0 & 1 & 2 & 3 & 4 \\
\hline$W_{k}$ & 1 & 0.93 & 1.15 & 1.07 & 1 \\
\hline${ }^{\mathrm{BZ}} d \quad[\AA]$ & - & 2.42 & 2.43 & 2.44 & 2.450 \\
\hline${ }^{\mathrm{AZ}} d$ & 2.359 & 2.37 & 2.37 & 2.38 & - \\
\hline${ }^{\mathrm{BZB}} d$ & - & - & 3.90 & 3.90 & 4.001 \\
\hline $\mathrm{AZA}_{d}$ & 3.852 & 3.88 & 3.98 & - & - \\
\hline${ }^{\mathrm{BZA}} d$ & - & 3.91 & 3.90 & 3.99 & - \\
\hline $\mathrm{ZBZ}_{d}$ & - & 3.95 & 3.97 & 3.98 & 4.001 \\
\hline${ }^{\mathrm{ZAZ}} d$ & 3.852 & 3.87 & 3.87 & 3.89 & - \\
\hline$\alpha(B: Z: B) \quad[\operatorname{deg}]$ & - & - & 106.7 & 106.9 & 109.47 \\
\hline$\alpha(\mathrm{A}: Z: A)$ & 109.47 & 109.7 & 114.3 & - & - \\
\hline$\alpha(B: Z: A)$ & - & 109.3 & 107.36 & 112.0 & - \\
\hline$<\mathrm{Vol}_{\text {B.centred }}>\left[\AA^{3}\right]$ & - & 7.28 & 7.37 & 7.41 & 7.55 \\
\hline$<\mathrm{Vol}_{\text {A.centred }}>$ & 6.74 & 6.84 & 6.86 & 6.91 & - \\
\hline$<\mathrm{Vol}_{\text {Z.sublattice }}>$ & 6.74 & 6.95 & 7.12 & 7.29 & 7.55 \\
\hline$<\mathrm{Vol}_{\text {Z.centred }}>$ & 6.74 & 6.95 & 7.07 & 7.28 & 7.55 \\
\hline |Difference | [\%] & 0.0 & 0.1 & 0.1 & 0.2 & 0.0 \\
\hline
\end{tabular}

\subsection{Model verification}

To confirm the validity of the model and its assumptions, we checked the quality of the model with its restricted free parameters.

1. Comparing the experimental distance-EXAFS points and error bars reported in the literature with model fit curves (see Fig. 2 (36 points with 10 free parameters) and the curves reported in [9-11]).

2. Comparing the «coordination number» curve predictions on the basis of SOP values obtained from distance-EXAFS measurement analysis, against independently measured coordination number values (see Fig. 3 and also [9-11]).

3. Checking for a correlation between the thermodynamic standard molar enthalpies of formation, $\Delta_{f} H^{0}, \mathrm{~kJ} / \mathrm{mol}$, of materials (Table 4 [10]) and the corresponding values obtained for the SOP coefficient.

The validity of the model with its restrictive assumptions is thus confirmed.

\section{FIR dielectric function $\varepsilon(\omega, x)$ for ternary zinc blende alloys}

The dielectric function

$$
\varepsilon(\omega)=\varepsilon_{\infty}+\sum_{j=1, n}\left\{S_{j} \omega_{j}^{2} /\left[\left(\omega^{2}-\omega_{j}^{2}\right)+i \omega \Gamma_{j}\right]\right.
$$

of phonon spectra of solids can be extracted from experimental reflectivity or transmission coefficients of a crystal, fitting the measured spectra via the Kramers-Kronig (KK) analysis. The KK output $\operatorname{Im}[\varepsilon(\omega)]$ directly yields the maxima for each oscillator line, assumed Lorentzian, with its three parameters $\left\{\omega_{j}, \Gamma_{j}\right.$, and $\left.S_{j}\right\}$, respectively, the frequency, the line half-

GaAsP
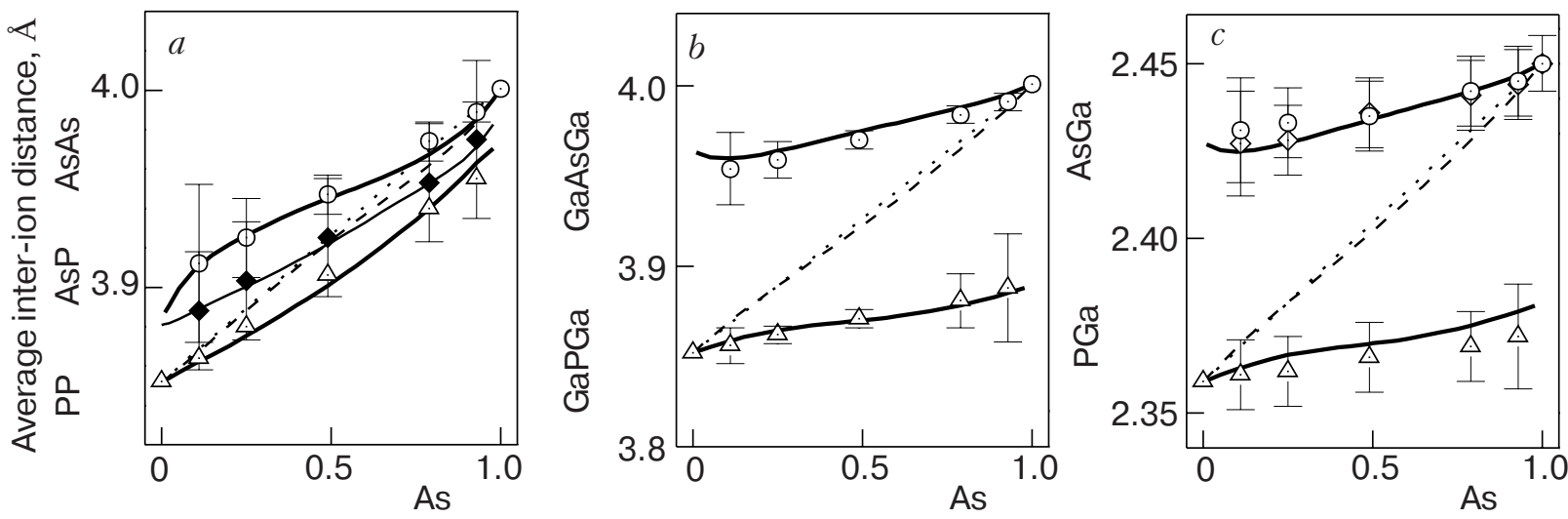

Fig. 2. Average inter-ion distances, $\AA$, as a function of relative content $x$ for $\operatorname{GaAs}_{x} \mathrm{P}_{1-x}$ [13]: comparison of model best fit curves versus reported experimental data. Points are (circles) for As-related (top curves), (triangles) for P-related (bottom curves), and (diamond) for mixed AsP ion distances. Linear combination of weighted average distances (LCWAD) curves (thin dashed lines) and corresponding reference Vegard law lines (thin dotted) are all reported [9]. 

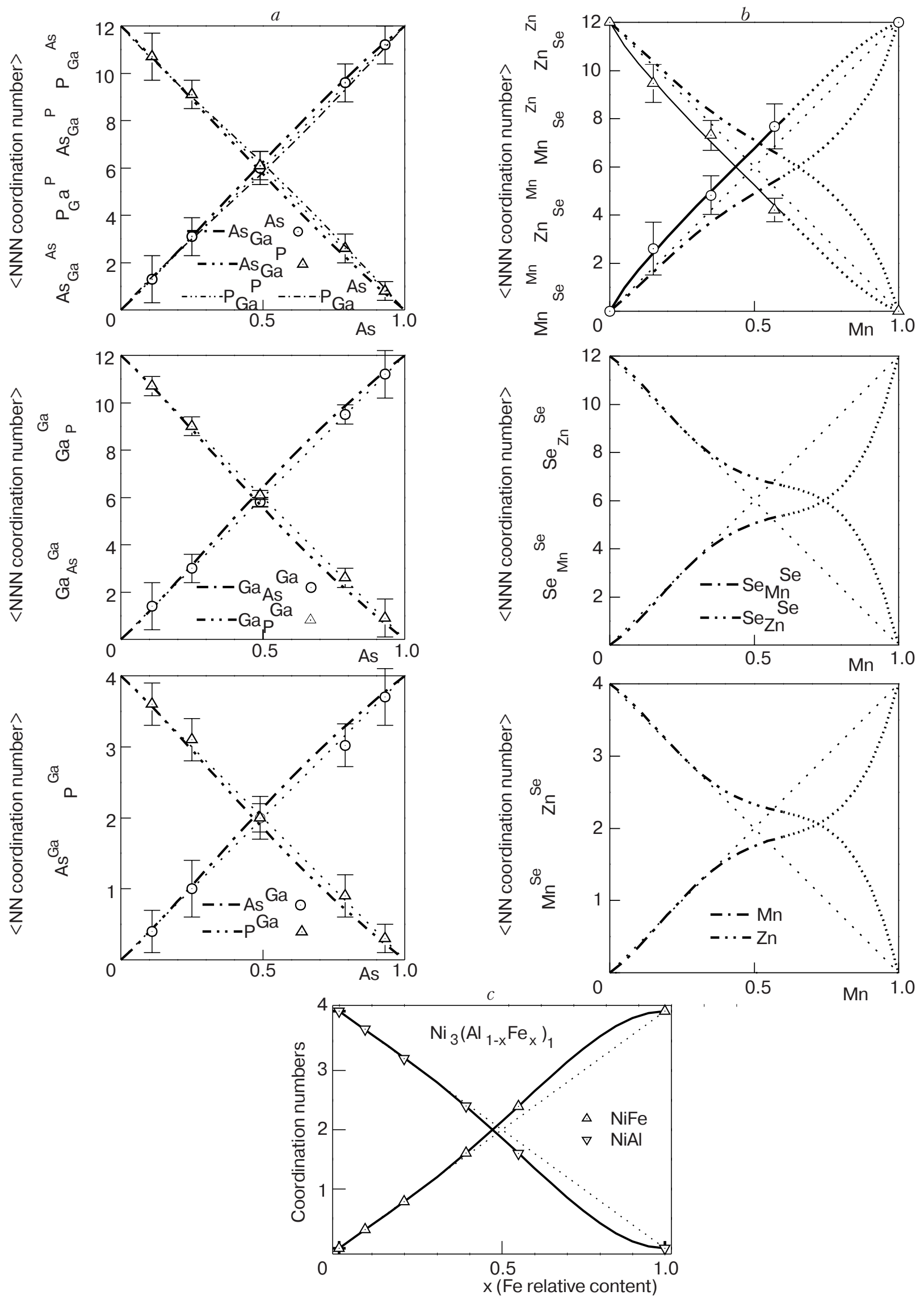

Fig. 3. Average coordination numbers as a function of relative content $x$ : comparison of model best fit curves using SOP values deduced from distance measurements [\{0.98,1.07,1.03\} $\operatorname{GaAs}_{x} \mathrm{P}_{1-x}$ [9] $(a) ;\{0.62,1.67,0\} \mathrm{ZnMn}_{x} \mathrm{Se}_{1-x}$ [10] (b); $\left.\{1.01,0.86,1.33\} \mathrm{Ni}_{3}\left(\mathrm{Al}_{1-x} \mathrm{Fe}_{x}\right)_{1}[11](c)\right]$ versus independently measured coordination number data. 
Table 4

Standard molar enthalpies of formation, $\Delta_{f} H^{0}, \mathrm{~kJ} / \mathrm{mol}$, of materials and corresponding SOP coefficients. Column $\left({ }^{\S}\right)$ gives origin and comments for reported SOP coefficient values derived from: $c-$ coordination number data, $d-$ distance data, $0^{*}$ - assumed $W_{3}=0$ value [10]

\begin{tabular}{|c|c|c|c|c|c|c|c|c|c|c|}
\hline Material & components & \multicolumn{3}{|c|}{$\Delta_{f} H^{0}, \mathrm{~kJ} / \mathrm{mol}$} & \multicolumn{5}{|c|}{ SOP } & \multirow[t]{2}{*}{$(\S)$} \\
\hline $\begin{array}{l}\text { ABZ } \\
\text { AYZ }\end{array}$ & $\begin{array}{l}\mathrm{AZ}+\mathrm{BZ} \\
\mathrm{AZ}+\mathrm{AY}\end{array}$ & \multicolumn{2}{|l|}{$\mathrm{AZ}$} & $\begin{array}{l}\mathrm{BZ} / \\
\mathrm{AY}\end{array}$ & $W_{0}$ & $W_{1}$ & $W_{2}$ & $W_{3}$ & $W_{4}$ & \\
\hline $\mathrm{ZnMnSe}$ & $\mathrm{ZnSe}+\mathrm{MnSe}$ & $-163.0^{a}$ & $<$ & $-106.7^{b}$ & 1 & 0.67 & 1.67 & 0.04 & 1 & $c$ \\
\hline & & & & & 1 & 0.62 & 1.70 & 0 & 1 & $d$ \\
\hline GaInAs & $\mathrm{GaAs}+\mathrm{InAs}$ & $-71.0^{a}$ & $<$ & $-58.6^{a, c}$ & 1 & 0.58 & 0.25 & 1.05 & 1 & $d$ \\
\hline GaAsP & $\mathrm{GaP}+\mathrm{GaAs}$ & $-88^{a}$ & $<$ & $-71.0^{a, c}$ & 1 & 0.93 & 1.15 & 1.07 & 1 & $d$ \\
\hline $\mathrm{ZnMnS}$ & $\mathrm{ZnS}+\mathrm{MnS}$ & $-206.0^{a},-205.98^{c}$ & $>$ & $-214.2^{a, c},-207.0^{b}$ & 1 & 1.78 & 0 & 0.01 & 1 & $d$ \\
\hline $\mathrm{ZnMnTe}$ & $\mathrm{ZnTe}+\mathrm{MnTe}$ & $-92.7^{a, c},-120.5^{b}$ & $<$ & $-94.7^{b}$ & 1 & 0.25 & 2.0 & 0.01 & 1 & $d$ \\
\hline CdMnTe & $\mathrm{CdTe}+\mathrm{MnTe}$ & $-102.5^{b}$ & $<$ & $-94.7^{b}$ & 1 & 0.68 & 1.33 & $0^{*}$ & 1 & $d+0^{*}$ \\
\hline
\end{tabular}

width, and the oscillator strength (OS). Note that while $\left\{{ }^{\mathrm{i} Z} \omega_{k}\right.$ and $\left.{ }^{\mathrm{i} Z} \Gamma_{k}\right\}$ are prime values, $\left\{{ }^{\mathrm{iZ}} S_{k}\right\}$ are sums over all the specific OSs $\left\{{ }^{\mathrm{iZ}} s_{k}\right\}$ multiplied by the relative ion-pair populations, taking into account ion-pair multiplicities (Eq. (4)), and by three SOP parameters $\left\{W_{1}, W_{2}, W_{3}\right\}$, which express the thermodynamics of the considered alloy. The introduction of SOPs links them to the OS of each ternary line. The sum

$$
\operatorname{Im}[\varepsilon(\omega, x)]=\sum_{j=1, n} \frac{S_{j} \omega_{j}^{2} \omega \Gamma_{j}}{\left(\omega^{2}-\omega_{j}^{2}\right)^{2}+\omega^{2} \Gamma_{j}^{2}}
$$

describes the total activity of all the oscillators over the frequency range considered. In zinc blende ternary $\mathrm{A}_{1-x} \mathrm{~B}_{x} \mathrm{Z}$ (or $\mathrm{AY}_{y} \mathrm{Z}_{1-y}$ ) compounds, each vibrating ion dipole pair $A Z$ and $B Z$ from each of the five elemental tetrahedron configurations $\left\{T_{k}\right\}_{k=0,4}$ contributes a phonon line to the spectrum (this idea was first presented by Verleur and Backer [14], who proposed a pioneering single-parameter model; the limits of the model were later discussed by us [15]). Thus $\operatorname{Im}[\varepsilon(\omega, x)]$ of the $\mathrm{A}_{1-x} \mathrm{~B}_{x} \mathrm{Z}$ spectra can be written as

$$
\begin{array}{cc}
\operatorname{Im}[\varepsilon(\omega, x)]=\frac{{ }^{\mathrm{AZ}} s_{0}{ }^{\mathrm{AZ}} \omega_{0}^{2}{ }^{\mathrm{AZ}} \Gamma_{0} \omega}{\left(\omega^{2}-{ }^{\mathrm{AZ}} \omega_{0}^{2}\right)^{2}+{ }^{\mathrm{AZ}} \Gamma_{0}^{2} \omega^{2}} P_{0}(x) & \text { binary AZ } \\
+\sum_{k=1,3}\left\{\frac{{ }^{\mathrm{BZ}} s_{k}{ }^{\mathrm{BZ}} \omega_{k}^{2 \mathrm{BZ}} \Gamma_{k} \omega}{\left(\omega^{2}-{ }^{\mathrm{BZ}} \omega_{k}^{2}\right)^{2}+{ }^{\mathrm{BZ}} \Gamma_{k}^{2} \omega^{2}}+\frac{(4-k)^{\mathrm{AZ}} s_{k}{ }^{\mathrm{AZ}} \omega_{k}^{2} \mathrm{AZ}_{\Gamma_{k} \omega}}{\left(\omega^{2}-{ }^{\mathrm{AZ}} \omega_{k}^{2}\right)^{2}+{ }^{\mathrm{AZ}} \Gamma_{k}^{2} \omega^{2}}\right\} P_{k}(x) & \text { ternary ABZ } \\
+\frac{{ }^{\mathrm{BZ}}{ }_{S_{4}}{ }^{\mathrm{BZ}} \omega_{4}{ }^{2}{ }^{\mathrm{BZ}} \Gamma_{4} \omega}{\left(\omega^{2}-\mathrm{BZ}_{\omega_{4}}\right)^{2}+{ }^{\mathrm{BZ}} \Gamma_{4}^{2} \omega^{2}} P_{4}(x) & \text { binary BZ }
\end{array}
$$

with the $P_{k}(x)$ 's defined in Eqs. (1).

Thus, the OS ${ }^{\mathrm{iZ}} S_{k}$ of each mode can be expressed by

$$
\begin{aligned}
{ }^{\mathrm{BZ}} S_{k}(x) & ={ }^{\mathrm{BZ}} s_{k} k P_{k}(x) \\
\text { and }{ }^{\mathrm{AZ}} S_{k}(x) & ={ }^{\mathrm{AZ}}{ }_{S_{k}}(4-k) P_{k}(x),
\end{aligned}
$$

with the specific $\mathrm{OS}{ }^{\mathrm{AZ}}{ }_{S_{0}}=\mathrm{AZ}_{S}$ and ${ }^{\mathrm{BZ}_{S_{4}}}=\mathrm{BZ}_{S}$ proper to the two binary constituents, and to $\left\{\mathrm{AZ}_{s_{k}}\right\}_{k=0,3}$ $\left\{{ }^{\mathrm{BZ}} s_{k}\right\}_{k=1,4}$ of the three ternary configurations.
If all four specific OSs for a given $\mathrm{iZ}$ pair are equal and independent of $x$, i.e.,

$$
\left\{{ }^{\mathrm{BZ}}{ }_{k}\right\}_{k=1,4}=\mathrm{BZ}_{s} \text { and }\left\{{ }^{\mathrm{AZ}}{ }_{s_{k}}\right\}_{k=0,3}=\mathrm{AZ}_{s}
$$

for a random distribution of $\mathrm{A}$ and $\mathrm{B}$ ions, the total OS of the respective modes $\mathrm{AZ}$ and $\mathrm{BZ}$ of $\mathrm{A}_{1-x} \mathrm{~B}_{x} \mathrm{Z}$ alloys is reduced identically to two linear functions of $x$, 

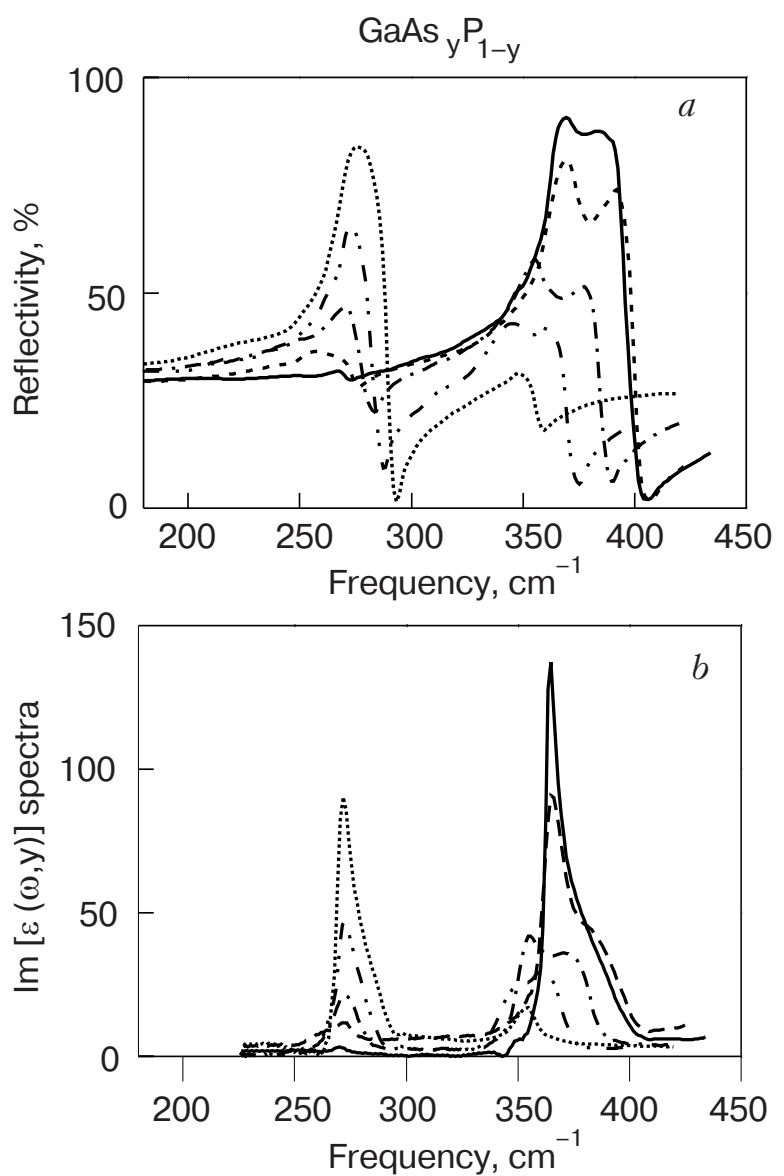

Fig. 4. $\mathrm{GaAs}_{y} \mathrm{P}_{1-y}$ : reflectivity [\%] spectra [14] (a); corresponding normalized $\operatorname{Im}[\varepsilon(\omega, y)]$ Kramers-Kronig derived spectra (b). Curves: $y=0.01$ (solid), 0.15 (dashed), 0.44 (dash-dotted), 0.72 (dash-dot-dotted), 0.94 (dotted).

$$
\begin{gathered}
\sum_{k=0,4}{ }^{\mathrm{BZ}} S_{k}(x)=4 x^{\mathrm{BZ}} s \\
\text { and } \sum_{k=0,4}{ }^{\mathrm{AZ}} S_{k}(x)=4(1-x)^{\mathrm{AZ}} s
\end{gathered}
$$

often referred to as the linear dependence on $x$.

To treat FIR spectra we make two FIR assumptions.

1. Specific OSs relative to a given ion pair is the same for all configurations,

$$
\left\{{ }^{\mathrm{BZ}} s_{k}\right\}_{k=1,4}={ }^{\mathrm{BZ}} s,\left\{{ }^{\mathrm{AZ}} s_{k}\right\}_{k=0,3}={ }^{\mathrm{AZ}} s .
$$

2. Analogously, we assume that for each of the two constituent ion pair populations, the line widths $\Gamma$ of any given composition spectrum are invariant:

$$
\left\{{ }^{\mathrm{AZ}} \Gamma_{k}\right\}_{k=0,3}={ }^{\mathrm{AZ}} \Gamma \text { and }\left\{{ }^{\mathrm{BZ}} \Gamma_{k}\right\}_{k=1,4}={ }^{\mathrm{BZ}} \Gamma .
$$

Thus to deconvolve a ternary spectrum with its 8 lines/spectrum, we have THREE SOP coefficients and TWO OS coefficients!

As was shown, the true tetrahedron populations in crystal lattices are determined by the alloy composition « $x$ » (or « $y$ » for $\mathrm{AY}_{y} \mathrm{Z}_{1-y}$ compounds) and the three SOP coefficients $\left\{W_{1}, W_{2}, W_{3}\right\}$.

To assess the credibility of the model FIR assumptions, a best-fit test is carried out to «derive» the two binary OS $\left\{{ }^{\mathrm{AZ}} s,{ }^{\mathrm{BZ}} s\right\}$ values from the $\mathrm{GaAs}_{y} \mathrm{P}_{1-y}$ spectra (Fig. 4) [14] that have a rich documentation in EXAFS [13], yielding SOP values.

Table 5

\begin{tabular}{|c|c|c|c|c|c|c|c|c|c|}
\hline \multirow{2}{*}{\multicolumn{2}{|c|}{$\begin{array}{l}\text { Phonon dipole } \\
\text { configuration }=\end{array}$}} & \multicolumn{4}{|c|}{ GaAs } & \multicolumn{4}{|c|}{$\mathrm{GaP}$} \\
\hline & & ${ }^{4} \mathrm{As}^{0} \mathrm{P}$ & ${ }^{3} \mathrm{As}^{1} \mathrm{P}$ & ${ }^{2} \mathrm{As}^{2} \mathrm{P}$ & ${ }^{1} \mathrm{As}^{3} \mathrm{P}$ & ${ }^{0} \mathrm{As}^{4} \mathrm{P}$ & ${ }^{1} \mathrm{As}^{3} \mathrm{P}$ & ${ }^{2} \mathrm{As}^{2} \mathrm{P}$ & ${ }^{3} \mathrm{As}{ }^{1} \mathrm{P}$ \\
\hline & $\mathrm{y}_{\mathrm{As}}$ & & & & & & & & \\
\hline \multirow[t]{5}{*}{$\omega\left[\mathrm{cm}^{-1}\right]$} & $1 \%$ & 257.8 & 261.9 & 264.3 & 268.4 & 363.5 & 365.5 & 377.0 & 379.0 \\
\hline & $15 \%$ & 259.6 & 261.6 & 263.6 & 271.2 & 362.9 & 364.9 & 366.9 & 381.0 \\
\hline & $44 \%$ & 265.1 & 269.2 & 271.2 & 273.2 & 350.7 & 352.7 & 363.6 & 373.9 \\
\hline & $72 \%$ & 269.7 & 271.7 & 273.7 & 279.6 & 342.8 & 344.8 & 350.9 & 360.6 \\
\hline & $94 \%$ & 269.5 & 271.5 & 278.5 & 280.5 & 345.8 & 347.8 & 349.8 & 351.8 \\
\hline \multirow[t]{5}{*}{$\Gamma\left[\mathrm{cm}^{-1}\right]$} & $1 \%$ & 8.64 & & & & 4.06 & & & \\
\hline & $15 \%$ & 10.04 & & & & 6.58 & & & \\
\hline & $44 \%$ & 10.74 & & & & 11.52 & & & \\
\hline & $72 \%$ & 6.00 & & & & 9.24 & & & \\
\hline & $94 \%$ & 3.99 & & & & 11.50 & & & \\
\hline \multirow{5}{*}{$\begin{array}{c}\text { A } \\
\text { (given } \\
\text { to two } \\
\text { decimals) }\end{array}$} & $1 \%$ & 0.00 & 0.00 & 0.00 & 0.04 & 1.84 & 0.05 & 0.00 & 0.00 \\
\hline & $15 \%$ & 0.02 & 0.01 & 0.08 & 0.17 & 1.05 & 0.55 & 0.09 & 0.01 \\
\hline & $44 \%$ & 0.13 & 0.21 & 0.28 & 0.12 & 0.26 & 0.57 & 0.42 & 0.11 \\
\hline & $72 \%$ & 0.52 & 0.46 & 0.18 & 0.02 & 0.02 & 0.13 & 0.31 & 0.26 \\
\hline & $94 \%$ & 1.63 & 0.28 & 0.02 & 0.00 & 0.00 & 0.00 & 0.03 & 0.17 \\
\hline
\end{tabular}

Individual deconvolution of $\mathrm{GaAs}_{y} \mathrm{P}_{1-y}$ spectra for each spectrum parameter $\left.\left\{{ }^{\mathrm{iGa}} \omega_{k},{ }^{\mathrm{iGa}} \Gamma\right\}_{i=\mathrm{As}, \mathrm{P} ; k=0,4}{ }^{\text {and }\left\{{ }^{\mathrm{GaAs}} s,{ }^{\mathrm{GaP}}\right.} s\right\}$ taken as free. $\left\{W_{1}=0.975, W_{2}=1.0715, W_{3}=1.034\right.$, as per $\mathrm{Wu}$ et al. data $\}$ : table of best fit parameter values for dipoles $\mathrm{GaAs}$ $k=1,4$ and for $\mathrm{GaP} k=0,3$. Amplitudes are given to two decimals. 


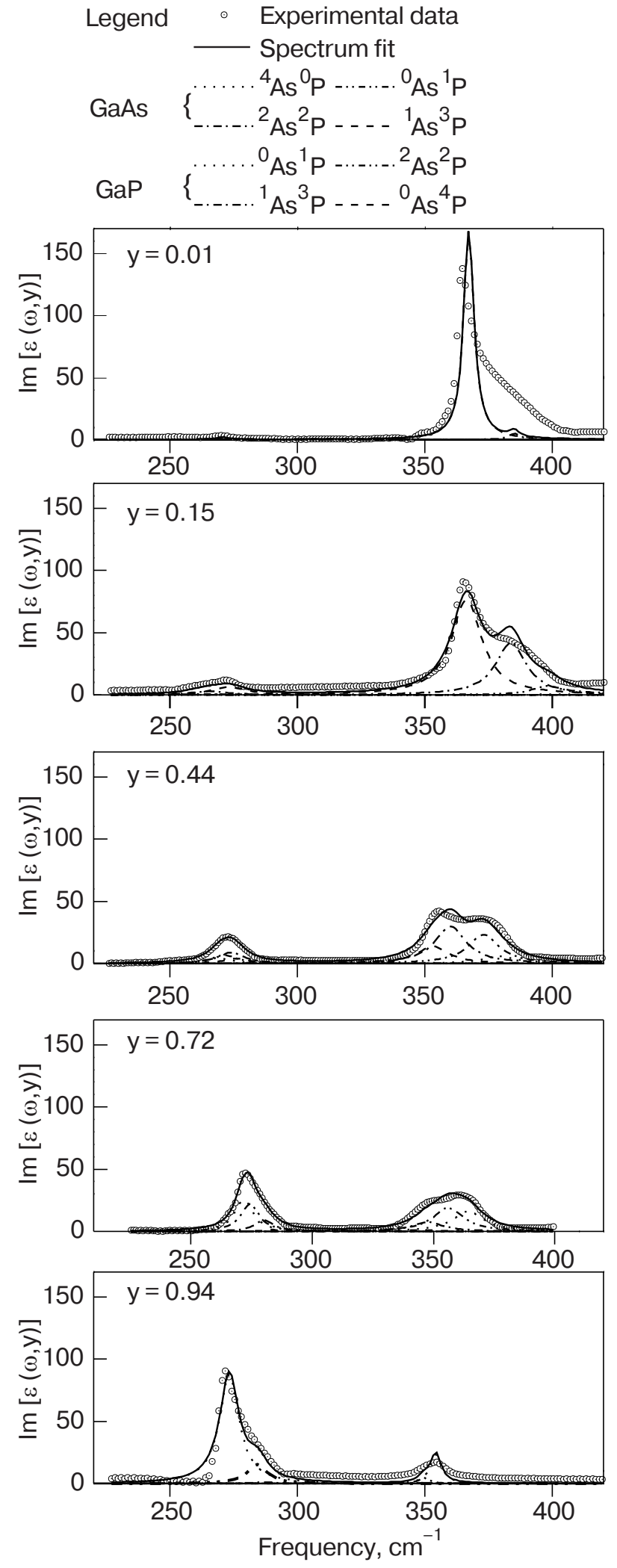

Fig. 5. Model best fit unfolding of $\mathrm{GaAs}_{y} \mathrm{P}_{1-y} \operatorname{Im}[\varepsilon(\omega, y)]$ spectrum for SOP coefficients $\left\{W_{1}=0.98, \quad W_{2}=1.07\right.$, $\left.W_{3}=1.03\right\}$. The four line bands of $\left\{{ }^{\mathrm{GaAs}} \omega_{k}\right\}_{k=1,4}$ and of $\left\{{ }^{\mathrm{GaP}} \omega_{k}\right\}_{k=0,3}$ are distinctly seen below and above $\omega=300 \mathrm{~cm}^{-1}$, respectively; experimental points (circles), best fit (solid lines), deconvolved lines (various discontinuous lines); the frequencies and intensities obtained are given in Table 5.

\section{Summary and conclusions}

The spectrum of any pure canonical, zinc blende ternary ABZ (or AYZ) material with its 5 tetrahedron configurations $\left\{T_{k}\right\}$ exhibits 8 phonon lines $(4 \mathrm{AZ}+4 \mathrm{BZ})$. The number of lines can be less than 8 when in pure defect-free materials extreme preferences prevent the formation of some configuration; this is observed with a transient element in $\mathrm{B}=\{\mathrm{Mn}, \mathrm{Fe}, \ldots\}[10,11]$ : ZnMnSe lacks one, ZnMnS lacks two; however, GaAlN also lacks one with another nearly evanescent [16]. Thus only 6, 4, 4 intense +2 weak phonon lines, respectively. More than 8 lines are observed when point defects occur (antisites, vacancies, ...), responsible for the extra lines (as reported for $\mathrm{HgCdTe}$ [17]). Thus FIR admits the detection of defects: vacancies, intersites, antisites, H-loading deformations, quantifying amount of impurity ions [18].

The statistical model of the optical dielectric function is applied to five $\mathrm{GaAs}_{y} \mathrm{P}_{1-y}$ (of type $\mathrm{AY}_{x} \mathrm{Z}_{1-x}$ ) FIR spectra [14] (see Fig. 4). In spite of the restricted number of parameters, the results show a good fit of the spectra (Fig. 5, Table 5); but most important, the best fit yields for ${ }^{\mathrm{GaAs}} s$ and ${ }^{\mathrm{GaP}} s$ values that overlap with published values within the uncertainty bars (Table 6).

Table 6

Individual unfolding of GaAs $\mathrm{P}_{1-y}$ spectra, $\left\{W_{1}=0.975\right.$, $\left.W_{2}=1.0715, W_{3}=1.034\right\}$ : table of best fit values $\left\{{ }^{1-9 A s} s_{y}\right.$, $\mathrm{GaP}_{s_{y}}$, their average values, and comparison to values in literature. An asterisk indicates unreliable low-signal values.

\begin{tabular}{c|c|c}
\hline \hline $\mathrm{y}_{\mathrm{As}}$ & $\mathrm{GaAs}$ & $\mathrm{GaP}$ \\
\hline $1 \%$ & $*$ & 1.97 \\
$15 \%$ & 2.18 & 1.98 \\
$44 \%$ & 1.68 & 2.49 \\
$72 \%$ & 1.58 & 2.77 \\
$94 \%$ & 2.00 & $*$ \\
All 5 together & 1.75 & 1.98 \\
(global values) & & $2.24 \pm 0.17$ \\
Aver. experimental & $1.84 \pm 0.11$ & $2.06 \pm 0.16$ \\
Aver. literature & $1.815 \pm 0.21$ & $1.15 \pm 0.17$ \\
ratio & $0.90 \pm 0.14$ &
\end{tabular}

Such a satisfactory reproduction validates the model assumptions and gives confidence the model is useful in giving a deeper understanding of the FIR results.

Equations, tables and figures taken from our previous publications (as referenced) are documented in greater detail.

Part of the work was supported by the EU TARIproject contract HPRI-CT-1999-00088. 
1. P.A. Lee, P.H. Citrin, P. Eisenberger, and B.M. Kincaid, Rev. Mod. Phys. 93, 769 (1981).

2. B.K. Teo, EXAFS: Basic Principles and Data Analysis, Springer Verlag (1986).

3. J.C. Mikkelsen and J.B. Boyce, Phys. Rev. B28, 7130 (1983).

4. A. Balzarotti, M.T. Czyzyk, A. Kisiel, N. Motta, M. Podgorny, and M. Zimnal-Starnawska, Phys. Rev. B30, 2295 (1984), ibid. 31, 7526 (1985).

5. A.S.Barker and J. Sievers, Rev. Mod. Phys. 47, 51 (1975).

6. D.W. Taylor, in: Dynamical Properties of Solids, vol. 2, G.H. Horton and A.A. Maradudin (eds.), North-Holland, N.-Y. (1975).

7. D.W Taylor, in: Optical Properties of Mixed Crystals, R.J. Elliot and I.P. Ipatova (eds.), Elsevier Science Publishers B.V. (1988), p. 35.

8. Sadao Adachi, Optical Properties of Crystalline Solids and Amorphous Semiconductors. Materials and Fundamental Principles, Kluver Academic Publishers, Boston (1999).

9. B.V. Robouch, A. Kisiel, and J. Konior, J. Alloys Compounds 339, 1 (2002).
10. B.V. Robouch, A. Kisiel, and J. Konior, J. Alloys Compounds 340, 13 (2002).

11. B.V. Robouch, E. Burattini, A. Kisiel, A.L. Suvorov, and A.G. Zaluzhnyi, J. Alloys Compounds 359, 73 (2003).

12. B.V. Robouch, E.M. Sheregii, and A. Kisiel, Phys. Status Solidi (in print)

13. Z. Wu, K. Lu, Y. Wang, J. Dong, H. Li, Ch. Li, and Zh. Fang, Phys. Rev. B48, 8694 (1993).

14. H.W. Verleur and A.S. Barker, Phys. Rev. 149, 715 (1966)

15. B.V. Robouch, A. Kisiel, and E.M. Sheregii, Phys. Rev. B64, 73204 (2001).

16. B.V. Robouch, A. Kisiel, I. Kutcherenko, and L.K. Vodopyanov, Appl. Phys. Lett. (submitted).

17. S.P. Kosyrev, L.K. Vodopyanov, R. Triboulet, Phys. Rev. B58, 1374 (1998).

18. A. Kisiel, B.V. Robouch, E. Burattini, A. Marcelli, M. Piccinini, M. Cestelli Guidi, P. Calvani, A. Nucara, E.M. Sheregii, J. Polit, and J. Cebulski, 5-th International Ural Seminar Radiation Damage Physics of Metals and Alloys, Book of abstracts, Snezhinsk, Russia (2003). 